\title{
MORBIDADE POR DOENÇAS RESPIRATÓRIAS EM PACIENTES HOSPITALIZADOS EM SÃO PAULO/SP
}

\author{
Marcos Tadashi Kakitani Toyoshima*, Gláucia Munemasa Ito, Nelson Gouvela \\ Trabalho realizado no departamento de Medicina Preventiva da Faculdade de Medicina da Universidade de São Paulo, São Paulo, SP.
}

*Correspondência

Rua Professor Antônio

Prudente, 41, apto. 506,

Liberdade, São Paulo, SP,

Cep $01509-010$

E-mail: $\quad$ mtkt@ terra.com.br

\begin{abstract}
RESUMO
OBjetivos. Analisar a morbidade por doenças respiratórias, medida pelas internações hospitalares, no município de São Paulo de 1995 a 2000, descrevendo o comportamento das internações por doenças respiratórias totais, pneumonias, asma e doença pulmonar obstrutiva crônica (DPOC), conforme sexo e idade, e identificar suas tendências.

Mitrodos. As informações referentes às internações hospitalares, para todas as idades e causas, foram obtidas através das Autorizações de Internações Hospitalares do SUS - Ministério da Saúde (DATASUS). Foi realizada análise descritiva com gráicos e tabelas.

Resultados. Houve decréscimo no número absoluto e na taxa de internações por doenças respiratórias totais em todas as idades, mas de maneira não uniforme em todo o período. Para DPOC, observou-se tendência de aumento importante, principalmente entre os idosos. A população masculina foi mais afetada do que a feminina em todos os anos, por quaisquer causas estudadas, com exceção da asma. As pneumonias representaram a causa mais importante de hospitalizaçôes, com 47\% das internações. As internações, principalmente das pneumonias, apresentaram padrão sazonal marcante.

Conclusia. Neste período de seis anos, nossas análises revelaram a participação importante das pneumonias como causa de internação. Porém, estas doenças vêm apresentando uma tendência decrescente, ao contrário de doenças crônicas como a DPOC e a asma. Estes resultados indicam uma ligeira mudança no perfil destas doenças no nosso meio, o que traz consequiências imediatas para o planejamento de serviços e para a formulaçãa de políticas de saúde.
\end{abstract}

Un ITERMOS: Morbidade. Doenças respiratórias. Epidemiologia. Tendências. Padrão sazonal.

\section{InTRODUÇÃO}

As doenças respiratórias constituem importante causa de adoecimento e morte em adultose crianças no mundo. Segundo dados da 0 rganização Mundial de Saúde (O MS), estas doenças representam cerca de $8 \%$ do total de mortes em países desenvolvidos e $5 \%$ em países em desenvolvimento ${ }^{1}$. A O MS estima ainda que, em 1995, 4,3 milhões de crianças com idade inferior a cinco anos morreram por doenças respiratórias agudas nos países em desenvolvimento, sendo a principal causa as pneumonias?

Estas doenças têm também um papel de destaque na mo rbidade da população, sendo freqüente causa de absenteísmo na escola e no trabalho, além de exercer enorme pressão sobre os serviços de saúde. Por exemplo, estima-se que cerca de 40 milhões de crianças menores de cinco anos adquirem pneumonia anualmente ${ }^{2}$ e que entre $5 \%$ a $15 \%$ dosadultosem países industrializadostêm doença pulmonar obstrutiva crônica (DPOC)

No Brasil, as doenças respiratórias agudas e crônicas também ocupam posição de destaque. Entre as principais causas de internação no Sistema Ú nico de Saúde - SUS, em 2001, estas doenças ocuparam o segundo lugar em freqüência, sendo responsáveis por cerca de $16 \%$ de todas as internações do sistema ${ }^{4}$.

Entretanto, apesar da importância epidemiológica das doenças respiratórias em nosso contexto, informações mais precisas sobre sua frequiência, distribuição e tendências de evolução recente ainda são escassas no Brasil, principalmente para morbidade, uma vez que para mortalidade dispomos de um Sistema de Informações (SIM) já bastante consolidado. Para suprir esta lacuna, o Ministério da Saúde vem disponibilizando informações sobre internações hospitalares por local de residência desde 1995, através do Sistema de Informações Hospitalares do Sistema Único de Saúde (SIH -SUS).

Este estudo teve como objetivo descrever as principais características da morbidade por doenças respiratórias no município de São Paulo, através do exame das internações hospitalaresno âmbito do SUS, que ocorreram neste município. Além disso, examinou-se em detalhe a evolução temporal deste grupo de doenças e de seus principais diagnósticos como as pneumonias (principal diagnóstico entre infecções respiratórias agudas), a asma eas D PO C (representantes das doenças respiratórias crônicas) no período de 1995 a 2000. Estas informações podem ser úteis para o planejamento de açõese para a gestão do sistema de saúde neste município.

\section{MÉTODOS}

As informações sobre as internações hospitalares por doenças respiratórias o corridas no município de São Paulo, no período de 1995 a 2000, foram obtidas de bancos de dados informatizados, disponibilizados pelo Ministério da Saúde, que contêm informações de todas as internações realizadas através das Autorizações de Internação Hospitalar (AlH) do Sistema Ú nico de Saúde (SUS). Estima-se que as internações no âmbito do SUS representam cerca de 60\% a 70\% do total de internações que ocorrem neste município. 
TOYOSHIMA MTK ET AL.

Tabela 1 - Distribuição das doenças respiratórias no município de São Paulo no período de 1995 a 2000, em número de internaçōes, em ambos os sexos e nas três faixas etárias

\begin{tabular}{|c|c|c|c|c|c|c|c|}
\hline Doenças & respiratórias & & DPOC & Asma & Pneumonias & Outras & Total \\
\hline \multirow[t]{3}{*}{ Número de internaçôes } & & $\mathrm{N}$ & 12066 & 25823 & 96772 & $\overline{72103}$ & $\overline{206764}$ \\
\hline & & $\%$ & $5,84 \%$ & $12,49 \%$ & $46,80 \%$ & $34,87 \%$ & - \\
\hline & Masculino & $\mathrm{N}$ & 6954 & 12781 & 53982 & 40562 & 114279 \\
\hline \multirow{5}{*}{ Sexo } & & $\%$ & $6,09 \%$ & $11,18 \%$ & $47,24 \%$ & $35,49 \%$ & - \\
\hline & Feminino & $\mathrm{N}$ & 5112 & 13041 & 42788 & 31539 & 92480 \\
\hline & & $\%$ & $5,53 \%$ & $14,10 \%$ & $46,27 \%$ & $34,10 \%$ & \\
\hline & 0.4 anos & $\mathrm{N}$ & 729 & 12328 & 52388 & 20053 & 85498 \\
\hline & & $\%$ & $0,85 \%$ & $14,42 \%$ & $61,27 \%$ & $23,45 \%$ & - \\
\hline \multirow[t]{4}{*}{ Faixa etária } & 5-64 anos & $\mathrm{N}$ & 5530 & 11817 & 30853 & 40118 & 88318 \\
\hline & & $\%$ & $6,26 \%$ & $13,38 \%$ & $34,93 \%$ & $45,42 \%$ & \\
\hline & 65 anos ou mais & N & 5805 & 1677 & 13523 & 11916 & 32921 \\
\hline & & $\%$ & $17,63 \%$ & $5,09 \%$ & $41,08 \%$ & $36,20 \%$ & - \\
\hline
\end{tabular}

0 s bancos de dados contêm informações relativas ao sexo e idade do paciente hospitalizado, seu diagnóstico principal, o tempo de hospitalização, a data de internação e a ocorrência de óbito durante a internação. Foram extraídas apenas informações de internações para

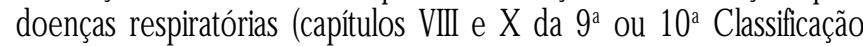
Internacional de Doenças - CID -95 e CID-106 respectivamente) que ocorreram no município de São Paulo no período especificado. Até 1996, as AlH continham os diagnósticos classificados pela CID-9 e a partir de 1997, pela CID-10.

Foram analisadas as doenças respiratórias em geral, assim como alguns dos diagnósticos mais comuns neste grupo de causas como pneumonia, DPO C e asma, nas faixas etárias de menor de cinco anos, entre 5 e 64 anos, e acima de 65 anos de idade.

Foram calculadas taxas de internações para cada diagnóstico por sexo e faixa etária, em cada ano, utilizando-se dados populacionais do município de São Paulo obtidos através da Fundação Sistema Estadual de Análise de Dados (SEADE) ${ }^{7}$ e do DATASUS ${ }^{8}$. A época da realização deste estudo, os dados do Censo 2000 ainda não estavam disponíveis.

As estimativas da população total e por sexo foram obtidas da Fundação SEADE, e por faixa etária, através da página do DATASUS na internet. N esta última, estavam disponíveis apenas os valores referentes aos anos de 1995, 1996 e 1998. A população para 0 ano de 1997 foi calculada por interpolação aritmética utilizando os dados de 1996 e 1998. As populações por faixa etária paraosanos de 1999 e 2000 foram estimadas a partir da taxa de crescimento médio dos anos anteriores. Pelo DATASUS, também não foi possível obter a população com idade superior a 64 anos. Assim, utilizamos dados da população com idade superior a 59 anos como denominador no cálculo das taxas de internações.

D evido ao pequeno número de anos estudados, decidiu-se por realizar apenas análises descritivas das características e das tendências das doenças respiratórias. Isto foi feito através do exame de gráficos de série temporal e de tabelas de freqüência.

\section{RESULTADOS}

Foram contabilizadas 206.764 internações por doenças respiratórias no município de São Paulo no período de 1995 a 2000
(Tabela 1). Dessas, as pneumonias representam a principal causa, correspondendo a aproximadamente $47 \%$ do total de internações respiratórias no perío do estudado. Asma e D POC participaram com, respectivamente, $12,5 \%$ e 5,8\% do total de internações. As demais do enças, responsáveis por cerca de $35 \%$ das internações respiratórias compreendem as infecções de vias aéreas superiores, doenças intersticiais pulmonares, pneumotórax, entre outras.

Houve pouca diferença na distribuição do número de internações por doenças respiratórias entre os sexos. Para D POC e para as pneumonias, houve maior número de internações de pacientes do sexo masculino do que do feminino, sendo que na asma, número ligeiramente superior foi observado do sexo feminino.

Analisando-se as internações respiratórias de acordo com as três faixas etárias estudadas, observou-se que as pneumonias levaram a um maior número de internações tanto entre os idosos quanto entre as crianças, sendo mais notável nesta última, atingindo a $61,3 \%$ de internações por doenças respiratórias nesta idade. Mesmo entre os adultos (5-64 anos) as internações por pneumonias superaram as por asma ou DPOC. As internações por asma se restringiram às crianças e adultos, enquanto a DPOC tem expressão maior entre os acima de 65 anos, onde representa cerca de 18\% das internações respiratórias (Tabela 1).

A média de idades dos pacientes internados foi de 57,7 anos para DPO C , 23,8 anos para pneumonias e 19 anos para asma. A fisio patologia e os fatores de risco explicam o motivo pelo qual a D POC obteve média de idade bem maior entre as doenças estudadas. Houve aumento da média de idades dos pacientes internados por DPOC, sendo de 50,7 anos em 1995 e 61,9 anos em 2000. Para a asma e para as pneumonias, as médias de idades se mantiveram.

Para D PO C, o tempo de hospitalização no perío do de 1995 a 2000 evoluiu de cerca de o ito dias para 11,2 dias, sendo que para as pneumonias, asma e para o total de doenças respiratórias o tempo de hospitalização se manteve praticamente inalterado.

Uma das informações disponíveis na AlH é se houve óbito do paciente durante a internação. Analisando estes dados, observamos que houve maior porcentagem de óbitos nas hospitalizaçõespor D POC, com $12,2 \%$ das internações evoluindo para morte. Das doenças estudadas, a asma é a que apresenta menor mortalidade (0,7\% de mortes). 
Tabela 2 - Taxa de internaçōes por doenças respiratórias (por 10,000 habitantes) no municipio de São Paulo, no período de 1995 a 2000

\begin{tabular}{|c|c|c|c|c|c|c|c|}
\hline Doenças respiratórias & 1995 & 1996 & 1997 & 1998 & 1999 & 2000 & $\Delta$ \\
\hline Todas & 41,74 & 35,05 & 30,52 & 32,06 & 33,22 & 31,08 & $-25,5 \%$ \\
\hline DPOC & 1,56 & 1,35 & 1,44 & 2,71 & 2,47 & 2,30 & $47,4 \%$ \\
\hline Asma & 4,20 & 4,39 & 4,02 & 4,14 & 4,42 & 4,24 & $0,9 \%$ \\
\hline Pneumonias & 21,93 & 16,34 & 13,49 & 14,58 & 15,06 & 13,99 & $-36,2 \%$ \\
\hline
\end{tabular}

Fonte: AIH/SUS e estimativas da populacãa residente, Fundacão SEADE.

¿: Variação das taxas de internaçôes do ano de 2000 comparadas com as do ano de 1995, em porcentagem.

Tabela 3 - Taxas de internaçōes (por 10 mil habitantes) por doenças respiratórias, conforme sexo, no município de São Paulo, no periodo de 1995 a 2000, com os respectivos resultados da análise de tendência

\begin{tabular}{|c|c|c|c|c|c|c|c|c|}
\hline Doença & Sexo & 1995 & 1996 & 1997 & 1998 & 1999 & 2000 & $\Delta$ \\
\hline Doenças respiratórias & $\begin{array}{l}\text { Masculino } \\
\text { Feminino }\end{array}$ & $\begin{array}{l}48,19 \\
35,79\end{array}$ & $\begin{array}{l}40,92 \\
29,65\end{array}$ & $\begin{array}{l}35,68 \\
25,78\end{array}$ & $\begin{array}{l}37,05 \\
27,49\end{array}$ & $\begin{array}{l}38,00 \\
28,85\end{array}$ & $\begin{array}{l}32,35 \\
29,68\end{array}$ & $\begin{array}{l}-32,9 \% \\
-17.1 \%\end{array}$ \\
\hline$D P O C$ & $\begin{array}{l}\text { Masculino } \\
\text { Feminino }\end{array}$ & $\begin{array}{l}1,89 \\
1,27\end{array}$ & $\begin{array}{l}1,62 \\
1,11\end{array}$ & $\begin{array}{l}1,79 \\
1,11\end{array}$ & $\begin{array}{l}3,32 \\
2,16\end{array}$ & $\begin{array}{l}2,91 \\
2,06\end{array}$ & $\begin{array}{l}2,50 \\
2,08\end{array}$ & $\begin{array}{l}32,3 \% \\
63,8 \%\end{array}$ \\
\hline Asma & $\begin{array}{l}\text { Masculino } \\
\text { Feminino }\end{array}$ & $\begin{array}{l}4,02 \\
4,37\end{array}$ & $\begin{array}{l}4,51 \\
4,29\end{array}$ & $\begin{array}{l}4,28 \\
3,78\end{array}$ & $\begin{array}{l}4,30 \\
3,99\end{array}$ & $\begin{array}{l}4,64 \\
4,21\end{array}$ & $\begin{array}{l}4,14 \\
4,35\end{array}$ & $\begin{array}{c}3,0 \% \\
-0,5 \%\end{array}$ \\
\hline Pneumonias & $\begin{array}{l}\text { Masculino } \\
\text { Feminino }\end{array}$ & $\begin{array}{l}25,84 \\
18,33\end{array}$ & $\begin{array}{l}19,32 \\
13,60\end{array}$ & $\begin{array}{l}15,74 \\
11,43\end{array}$ & $\begin{array}{l}17,01 \\
12,35\end{array}$ & $\begin{array}{l}17,30 \\
13,01\end{array}$ & $\begin{array}{l}14,62 \\
13,30\end{array}$ & $\begin{array}{l}-43,4 \% \\
-27,4 \%\end{array}$ \\
\hline
\end{tabular}

Fonte: AIH/SUS e estimativas da população residente, Fundação SEADE.

$\Delta$ : Variação das taxas de internaçōes do ano de 2000 comparadas com as do ano de 1995, em porcentagem.

Tabela 4 - Taxas de internaçōes (por 10 mil habitantes) por doenças respiratórias, conforme faixa etária, no município de São Paulo, no período de 1995 a 2000

\begin{tabular}{|c|c|c|c|c|c|c|c|c|}
\hline Doenças respiratórias & Faixa etária & 1995 & 1996 & 1997 & 1998 & 1999 & 2000 & $\Delta$ \\
\hline \multirow{3}{*}{ Todas } & 1 & 183,38 & 190,39 & 153,46 & 164,73 & 172,62 & 172,35 & $-6,0 \%$ \\
\hline & $\|$ & 41,81 & 34,78 & 31,70 & 33,19 & 34,59 & 31,15 & $-25,5 \%$ \\
\hline & III & 132,35 & 107,97 & 104,32 & 108,20 & 110,69 & 99,31 & $-25,0 \%$ \\
\hline \multirow[t]{3}{*}{$D P O C$} & I & 2,02 & 2,62 & 1,23 & 0,75 & 0,99 & 1,16 & $-42,6 \%$ \\
\hline & $\|$ & 0,95 & 0,72 & 0,83 & 1,56 & 1,37 & 1,25 & $31,6 \%$ \\
\hline & III & 6,94 & 6,64 & 7,88 & 16,61 & 15,36 & 14,53 & $109,4 \%$ \\
\hline \multirow[t]{3}{*}{ Asma } & 1 & 19,22 & 25,57 & 24,58 & 26,33 & 26,53 & 28,43 & $47,9 \%$ \\
\hline & $\|$ & 2,49 & 2,53 & 2,28 & 2,24 & 2,53 & 2,23 & $-10,4 \%$ \\
\hline & III & 3,53 & 3,59 & 2,76 & 3,13 & 3,79 & 2,94 & $-16,7 \%$ \\
\hline \multirow[t]{3}{*}{ Pneumonias } & I & 124,25 & 116,00 & 90,16 & 98,15 & 104,02 & 100,84 & $-18,8 \%$ \\
\hline & $\|$ & 8,99 & 6,21 & 5,25 & 5,71 & 5,90 & 5,23 & $-41,8 \%$ \\
\hline & |l| & 32,37 & 24,68 & 25,23 & 27,07 & 26,27 & 23,67 & $-26,9 \%$ \\
\hline
\end{tabular}

Fonte: AIH/SUS e estimativas da população residente, DATASUS.

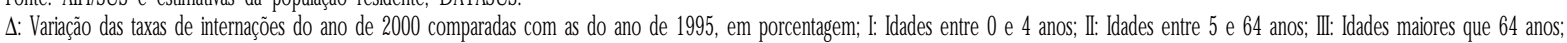

Analisando a tendência temporal das internações respiratórias entre 1995 e 2000, observou-se uma redução de cerca de 26\% nataxa de internação para doenças respiratórias totais, em grande parte devida à diminuição marcante das internações por pneumonias. Por outro lado, enquanto as internações por asma se mantiveram praticamente constantes, as internações por DPOC aumentaram substancialmente no período (Tabela 2).

Comparando-se a evolução das doenças respiratórias para as populações masculina e feminina, observamos uma taxa de internações maior na população masculina do que na feminina em todos os anos estudados, assim como para todas as causas de internação estudadas, com exceção da asma. Houve tendência de diminuição das taxas por doenças respiratórias em geral e por pneumonias para ambos os sexos, mas sendo mais predominante no sexo masculino. Para asma e DPOC, a tendência de aumento observada foi semelhante para ambos sexos (Tabela 3).

$\mathrm{N}$ a análise entre as diferentes faixas etárias (Tabela 4), observase que, apesar das internações por D PO C aumentarem de maneira acentuada, principalmente entre os maiores de 65 anos, e das internações por asma aumentarem entre as crianças, a tendência geral para as internações respiratórias to tais é de redução, principalmente devido às pneumonias, que apresentam tendência decrescente em todas as faixas etárias, com destaque para as crianças menores de cinco anos. 


\section{Figura 1 - Número de internaçōes por doenças respiratórias no municipio de São Paulo em cada mês, no perído de 1995 a 2000.}

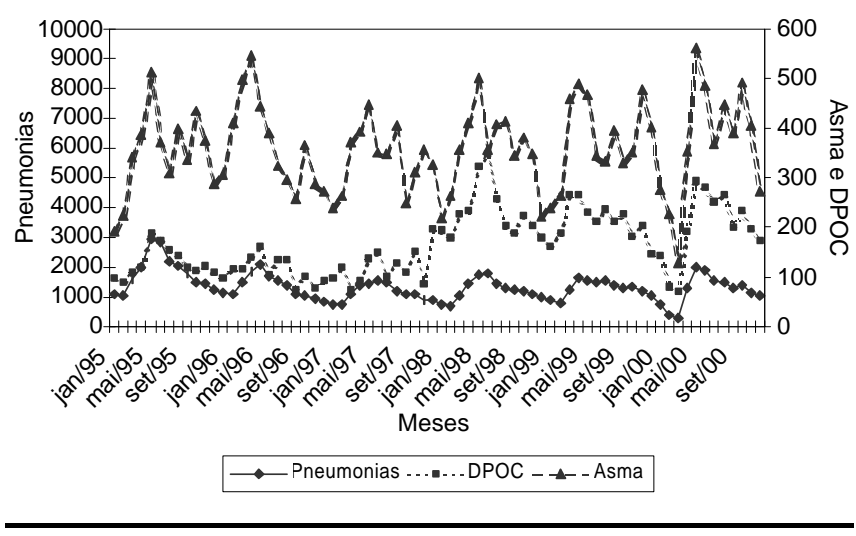

$\mathrm{Na}$ análise do padrão sazonal do número de internações por doenças respiratórias em geral, observa-se padrão típico para estas doenças em todos os anos: aumento do número de internações no primeiro semestre e diminuição no segundo. Os picos sempre se encontram em meses mais relacionados ao inverno e os vales naqueles mais relacionados ao verão. 0 mesmo padrão foi observado para as internações por pneumonias e por asma. Porém, para as internações por DPOC, este padrão sazonal não é tão marcante (Figura 1).

\section{Discussão}

Este trabalho procurou analisar as características principais da morbidade por doenças respiratórias no município de São Paulo e suas tendências no perío do de 1995 a 2000, utilizando dados do Sistemade Informações Hospitalares (SIH) do SUS, através de suas Autorizações de Internação Hospitalar (AlH).

U ma série de cuidados deve ser tomada ao interpretar os resultados apresentados. Primeiro, é preciso lembrar que este sistema de informações foi desenhado para fins administrativos e não para investigações epidemiológicas, o que poderia comprometer a qualidade das informações. Um outro problema é a possibilidade de viés de seleção. Hospitais não conveniados ao SUS, que, portanto, não emitem AlH, não foram incluídos neste estudo. 0 u seja, parcelas da população que são economicamente mais favorecidas estão sub-representadas.

Todavia, apesar da AlH ser um instrumento administrativo, alguns estudos têm demonstrado a confiabilidade de suas informações para estudos epidemiológicos. Estudos da validação das informações constantes daAlH mostram que existe um grau muito grande de concordância para diagnósticos usando três dígitos (82,5\%) e uma confiabilidade ainda maior para as variáveis demográficas, quando em comparação aos exames de prontuários. ${ }^{9}$ Além disso, estima-se que cerca de $70 \%$ dos leitos hospitalares em São Paulo pertencem a este sistema.

Além disso, houve alteração da Classificação Internacional de D oenças (CID) durante o período estudado. Isso pode ter influenciado e até dificultado o preenchimento do diagnóstico da doença pelos profissionais dasaúde. Por fim, utilizamosneste estudo taxas de hospitalização, po rém, é preciso lembrar que essastaxas podem incluir um mesmo paciente mais de uma vez no numerador. Desse modo, deve-se ter em mente que a taxa de internações de cada doença não reflete necessariamente a prevalência ou a incidência dessas morbidades na população.

De todo modo, foi possível observar que houve uma tendência de diminuição das internações por doenças respiratórias no período analisado, principalmente entre a população do sexo masculino. A principal razão para essa tendência foi i diminuição nas internações por pneumonias, que apresentaram queda acentuada entre 1995 e 2000. Por outro lado, doenças crônicas como a asma em criançase a DPOC em idosos apresentaram aumento de internações. Porém, como as pneumonias são responsáveis pela maior parte das internações respiratórias, a tendência geral foi de queda.

Todavia, estas variações não ocorreram de maneira uniforme no período. A partir de 1998 houve um pequeno aumento na taxa de internações respiratórias totais. $\mathrm{N}$ um exame mais detalhado, pode-se notar que isto foi devido principalmente às internações por D POC, que mudaram substancialmente de patamar a partir de 1998, muito provavelmente devido à introdução da nova codificação pelaC ID-10 naquele ano.

A queda nas internações por doenças respiratórias totais, ou mais especificamente por pneumonias pode ter ocorrido por vários motivos. Primeiro, esta tendência pode ter apenas acompanhado o movimento mais geral das internações no âmbito do SUS como um todo. Por outro lado, pode-se especular que tem havido melhora na atenção primária e muitas pneumonias ago ra estão sendo tratadas ambulatorialmente. Pode ter havido ainda melhora em determinadas circunstâncias que são conhecidos fatores de risco para pneumonias, como a aglomeração domiciliar, a freqüência em creches, o tabagismo dos pais, a instrução da mãe, a poluição ambiental, e variáveis tais quais peso ao nascer, desnutrição e desmame precoce. ${ }^{10}$ De todo modo, estudos mais detaIhados sobre as razões das alterações nas taxas de ho spitalização serão de grande importância para o planejamento futuro de ações de saúde.

0 aumento importante observado para as internações por D POC, principalmente entre aqueles acima de 65 anos, pode ser explicado pelo próprio envelhecimento da população e o maior tempo de exposição ao tabagismo com o passar dos anos. Houve, ainda, aumento do número de internações por D POC em ambos os sexos, sendo mais acentuado na população feminina, o que pode ser explicado por aumento da incidência de tabagismo entre as mulheres. Assim como em nosso estudo, a DPOC também está se tornando cada vez mais prevalente em mulheres de vários países, tais como Índia, México, Cuba, Egito, África do Sul e China ${ }^{11}$.

Assim como para as pneumonias, medidas de controle da poluição ambiental são importantes para diminuição da prevalência de D POC. Porém, a principal estratégia deve enfocar campanhas e medidas individuais que desestimulem a população ao tabagismo, já que este é 0 principal fator de risco à doençad ${ }^{12,13}$.

Assim como a D PO C, houve aumento nas taxas de internação por asma, mas apenas na população menor de cinco anos. A educação ambiental e maior cobertura vacinal são fatores que podem diminuir a incidência de asma, principalmente em crianças. Isto porque a infecção de vias aéreas superiores, pêlo de animais, ácaros e tabagismo, entre outros fatores, são importantes desencadeadores de crises asmáticas e muitas delas podem ser prevenidas, evitando internações ${ }^{14,15}$. 
Nosso estudo revelou também um padrão sazonal para as internações por asmae pneumonia, sendo maior nos meses relacionados ao outono ou inverno. A maioria dos estudos que mostra o padrão sazonal se localiza em países de clima temperado, em que as estações do ano são mais definidas. $N$ a Finlândia, por exemplo, as grandes diferenças climáticas entre verão e inverno têm importante papel no padrão sazonal das hospitalizações tanto para pneumonias quanto para DPOC, com maior número de internações durante 0 inverno ${ }^{16,17}$.

Para a asma, observamos flutuação no número de internaçõos no decorrer dos meses, com picos principalmente entre março e maio (meses de outono) e mínimo em janeiro (verão). Vários estudos demonstraram a mesma tendência, principalmente na faixa etária pediátrica ${ }^{18,19,20}$. Na Grécia e em Malta (países mediterrâneos), foram descritas variações sazonais associadas ao tempo frio e úmido, com diferenças entre subgrupos etários na infância ${ }^{21,22}$.

Apesar de se tratar de um estudo descritivo, as informações aqui analisadas podem indicar tendências recentes nas taxas de internações hospitalares por doenças respiratórias no municííio de São Paulo. Cabe lembrar 0 curto período de tempo analisado, que dificulta uma análise mais precisa dessas tendências. Porém, dados completos sobre hospitalização pelo SUS através das AlHs só puderam ser disponiveis a partir de 1995. Assim, é possível que estudos de internações por doenças respiratórias, analisando um tempo maior, possam trazer resultados mais precisos. De maneira geral, estes resultados esboçam uma mudança no perfil da importância das doenças respiratórias no nosso meio , o que traz consequêencias imediatas e futuras para o planejamento de serviços e para a formulação de políticas de saúde.

Conflito de interesse: Subvencionado pela Fundação de Amparo à Pesquisa do Estado de São Paulo (FAPESP) (Projeto 00/11724-0).

\section{SUMMARY}

TrENDS IN MORBDITY FOR RESPRATORY DISEASES AMONG
HOSPTALIZED PATIENTS IN THE CITY OF SÁO PAULO

0 BJECTIVES. To analyzetrends in morbidity dueto respiratorydiseases, using hospital admissions in the city of São Paulo from 1995 to 2000. To describe the evolution of themorbidity for respiratorydiseasesin general and for pneumonias, for asthma and for chronic obstructive pulmonary disease (CO PD), bysex and age.

Metr ODS. The information on hospitaladmissions forall ages and causes were obtained from the H ospitalizations Information System ofthe Brazilian $M$ inistry of H ealth (DATASUS). Descriptive analysis of patterns and trends were performed.

RESULTS. There wasa decreasingtrend in the absolute number and the rate of hospitalization for respiratory illnesses for all ages but not uniformly duringthe period. For CO PD, anincreasingtrend was observed, especiallyfor the elderly. The male population was more affected than the female in all years, for any studied causes with the exception of asthma. The pneumonias represented the most important cause of hospitalizations, with $47 \%$ of all respiratoryadmissions. Important seasonal pattern wasobserved for pneumonia admissions.

CONCLUSION. In this period of 6 years, our analyses had disclosed an important participation of pneumonias asa cause for respiratoryadmissions. H owever, thesediseases presented a decreasingtrend, in contrast to chronic illnesses such as CO PD and asthma. These results point out to a changing pattern in the respiratory morbidity with direct consequences for public health policy making. [Rev Assoc Med Bras 2005; 51(4): 209-13]

\section{KEY WORDS: Morbidity. Respiratory diseases. Epidemiology. Trends.} Seasonal trends.

\section{REFERENCIAS}

1. W orld H eath O rganization. 1997:W orld H ealth Report. Geneva. WHO O 1998.

2. World Health O rganization. 1995: The Division of Diarrhoeal and Acute Respiratory Disease Control. URL:http://www.who.int/chd/publications/ cdd/pofact77.htm.

3. Anto JM, Vermeire $P$, Vestbo J, Sunyer J. Epidemiology of chronic obstructive pulmonary disease. Eur Respir J 2001;17(5):982-94.

4. Ministério da Saúde. Anuário Estatístico de Saúde do Brasil - 2001. U RL: http:/ /portal.saude.gov.br/saude/aplicacoes/anuario 2001

5. O rganização Mundial de Saúde. Classificação Internacional de Doenças Revisão 1975. Centro da O MS para Classificação de D oenças em Português - Ministério da Saúde/ U niversidade de São Paulo, 1985.

6. O rganização Mundial de Saúde. CID-10/ O rganização Mundial de Saúde; tradução - Centro Colaborador da O MS para a C lassificação de D oenças em Português. $3^{\text {a }}$. edição. São Paulo: Editora daU niversidade de São Paulo, 1996.

7. Fundação Sistema Estadual de Análise de Dados (Fundação SEADE). Informações dos Municípios Paulistas (São Paulo em Dados). http:// www.seade.gov.br/cgi-bin/homev98/seade_op.ksh

8. Sistema Ú nico de Saúde - Ministério da Saúde - Brasil. Sistema de Informações Hospitalares do SUS (SIH/SUS). http://tabnet.datasus. gov.br/ cgi/deftohtm.exe?sih/cnv/mrsp.def

9. Veras CMT, Martins MAS. Confiabilidade dos dados nos formulários de Autorização de Internação H ospitalar (AlH ).Cad SaúdePubl 1994;10(3):339355.

10. Graham N MH. The epidemiology of acute respiratory infections in children and adults: a global perspective. Epidemiologic Reviews1990;12:149-78.

11. Lomas DA, Silverman EK. The genetics of chronic obstructive pulmonary disease. Respir Res2001;2(1):20-6.

12. Pattwels RA, Buist AS, Calvelry PMA, Jenkins CR, H urd SS. G lobal strategy for the diagnosis, management, and prevention of chronic obstructive lung disease. N HI.BI/W HO Global Initiative for Chronic O bstructive Lung D isease (GO LD) W orkshop summary. Am J Respir Crit Care M ed 2001;163:1256-1276.

13. Ward SA, Casaburi R. $21^{\text {st }}$ Century Perspective on Chronic Obstructive Pulmonary D isease. Respiration 2001;68:557-561.

14. Burney PGJ. Strategy for asthma. Br M ed J 1991;303:571-573.

15. N ational Institutes of $\mathrm{H}$ ealth. G lobal Initiative for Asthma - Pocket $\mathrm{G}$ uide for Asthma Management and Prevention. 1998.

16. Saynajakangas $P$, Keistinen $T$, Tuuponen $T$. Seasonal fluctuations in hospitalisation for pneumonia in Finland. Int J Circumpolar Health 2001;60(1):34-40.

17. Vilkman S, Keistinen T, Tuuponen T, Kivela SL. Seasonal variation in hospital admissions for chronic obstructive pulmonary disease in Finland. Arctic M ed Res 1996;55(4):182-6.

18. Dales RE, Schweitzer I, Toogood JH, et al. Respiratory infections and the autumn increase in asthma morbidity. Eur Respir J 1996;9(1):72-7.

19. Harju T, Keistinen T, Tuuponen T, Kivela SL. Seasonal variation in childhood asthma hospitalisations in Finland, 1972-1992.EurJ Pediatr 1997;156(6):4369.

20. Khot A, Burn R, Evans $N$, et al. Seasonal variation and time trends in childhood asthma in England and Wales 1975-81. Br Med J (Clin Res Ed) 1984;289(6439):235-7.

21. Grech V, Balzan M, Asciak RP, Buhagiar A. Seasonal variations in hospital admissions for asthma in Malta. J Asthma 2002;39(3):263-8.

22. Priftis K, Anagnostakis J, H arokoposE, et al. Time trends and seasonal variation in hospital admissions for childhood asthma in the Athens region of G reece: 1978-88. Thorax 1993;48(11):1168-9.

Artigo recebido: 12/03/04

Aceito para publicação: 21/05/04 